\title{
Wenn Schweigen gefährlich ist: „Speaking-up“ bei Sicherheitsbedenken
}

When silence is dangerous: "Speaking-up" about safety concerns

David LB Schwappach ${ }^{\mathrm{a}, \mathrm{b}, \dagger}$

\author{
${ }^{a}$ Swiss Patient Safety Foundation, Asylstrasse 77, 8032 Zurich, Switzerland \\ ${ }^{\mathrm{b}}$ Institute of Social and Preventive Medicine (ISPM), University of Bern, Finkenhubelweg 11, \\ 3012 Bern, Switzerland. schwappach@patientensicherheit.ch \\ $\dagger$ Corresponding author;
}




\section{Abstract}

\section{Background:}

5 Open and direct communication („speaking-up“) about errors, bypassed safety rules and risky

6 behaviours among hospital staff is required to avoid patient harm and is an essential

7 characteristic of an established safety culture. In German-speaking countries, little is known

8 about speaking-up behaviours among health care professionals (HCP) in hospitals.

9 Method: Safety concerns and speaking-up behaviours among HCP of nine oncological units of 10 eight hospitals were assessed using a self-administered survey. A vignette was embedded to assess hypothetical speaking-up and its predictors. The association of hierarchical position and speaking-up was investigated. 1'013 physicians and nurses completed the survey (65\% response rate).

Results:

$53 \%$ of HCP reported to have concerns for patient safety at their unit, „sometimes, „frequently“ or „very frequently“. Colleagues bypassing important safety rules at least "sometimes” was reported by $30 \%$. A considerable fraction of responders reported episodes of withholding voice. Nearly $20 \%$ said they did not communicate safety problems at their unit at least sometimes. $73 \%$ among staff with higher and $60 \%$ among those with lower hierarchical position said they had never withheld information which could have reduced threats to patients $(O R=1.8$, $p \leq 0.001)$. Many responders felt that speaking-up is often difficult and challenging. $32 \%$ responded that they would not speak-up about a missed hand-disinfection towards a colleague who assesses the wound of a recently operated oncological patient.

Conclusions: HCP in hospital frequently experience safety concerns and often withhold them. An important resource for better patient safety is lost. The development of interventions to improve speaking-up culture is warranted.

Keywords: patient safety; medical errors; communication; speaking-up; oncology 


\section{Zusammenfassung}

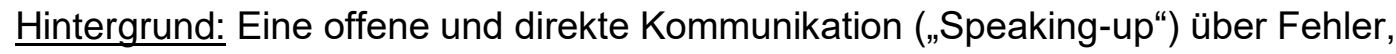
übergangene Sicherheitsregeln und risikoreiche Verhaltensweisen zwischen Mitarbeitenden im Krankenhaus ist zentral, um Patienten vor Schäden zu schützen und ein wesentlicher Aspekt einer etablierten Sicherheitskultur. Bislang ist im deutschsprachigen Raum wenig über das Speaking-up Verhalten von Mitarbeitenden im Krankenhaus bekannt.

Methode: In einer schriftlichen Befragung untersuchten wir Sicherheitsbedenken und Speakingup Verhalten unter Mitarbeitenden neun onkologischer Abteilungen von acht Krankenhäusern. Mit einer Fallgeschichte untersuchten wir Prädiktoren für das hypothetische Speaking-up. Der Zusammenhang zwischen Speaking-up und hierarchischer Position wurde analysiert. 1'013 Ärztinnen, Ärzte und Pflegefachpersonen beteiligten sich (Rücklauf: 65\%). Ergebnisse: 53\% der befragten Fachpersonen gaben an, „manchmal“, „häufig“ oder „sehr häufig“ konkrete Bedenken zur Patientensicherheit auf ihrer Abteilung zu haben. Die NichtEinhaltung von wichtigen Sicherheitsregeln durch Kollegen berichteten 30\% „manchmal“ und $13 \%$ „häufig“ oder „sehr häufig“. Das Zurückhalten von Sicherheitsbedenken wurde von einem substantiellen Anteil der Befragten berichtet. Knapp 20\% berichteten, mindestens „manchmal“ mögliche Sicherheitsprobleme in ihrer Abteilung nicht anzusprechen. Unter den Personen mit Leitungsfunktion haben $73 \%$ noch nie Hinweise verschwiegen, die möglicherweise eine Gefahr für Patienten reduziert hätten, während es unter den Personen ohne Leitungsfunktion 60\% waren $(\mathrm{OR}=1.8, \mathrm{p} \leq 0.001)$. Das Speaking-up empfindet ein erheblicher Teil der Befragten als schwierig und herausfordernd. 32\% der Befragten gab an, dass sie einen Kollegen nicht auf die ausgelassene Hände-Desinfektion bei einer frisch-operierten onkologischen Patientin hinweisen würden.

Schlussfolgerungen: Mitarbeitende im Spital haben häufig Sicherheitsbedenken, sprechen diese aber oft nicht an. Dadurch geht ein wichtiges Potential zur Verbesserung der Patientensicherheit verloren. Die Entwicklung von Interventionen zur Förderung der Speakingup Kultur ist eine wichtige zukünftige Aufgabe um diese Ressource zu nutzen. 
Schlüsselwörter: Patientensicherheit; Medizinische Fehler; Kommunikation; Speaking-up;

Onkologie

\section{Einleitung}

Die offene und respektvolle Kommunikation über Fehler, übergangene Sicherheitsregeln und risikoreiche Verhaltensweisen zwischen Mitarbeitenden im Krankenhaus ist zentral, um Patienten vor Schäden zu schützen, und ein wesentlicher Aspekt einer etablierten

Sicherheitskultur. Viele Sicherheitsmassnahmen bauen auf die Aufmerksamkeit und Kommunikation der Beteiligten, wenn etwas nicht nach Plan läuft. So kann beispielsweise die chirurgische Checkliste nur erfolgreich sein, wenn Mitarbeitende im OP Auffälligkeiten und Unstimmigkeiten sofort ansprechen. Analysen von Zwischenfällen zeigen jedoch, dass Abbrüche und Versagen in der Kommunikation in kritischen Momenten häufig vorkommen und eine Kernursache dafür sind, dass Fehler nicht rechtzeitig abgefangen werden [1]. Studien vor allem aus dem anglo-amerikanischen Raum dokumentieren, dass Gesundheitsfachpersonen ihre Bedenken zur Patientensicherheit häufig vollständig zurückhalten oder allenfalls vorsichtig, teilweise, uneindeutig oder zurückhaltend vorbringen, obwohl das „Speaking-up“ erforderlich wäre [2;3]. Speaking-up bezeichnet die verbindliche Kommunikation von Sicherheitsbedenken durch Informationen, Fragen, Einschätzungen oder Meinungsäusserungen in klinischen Situationen, in denen es akuten Handlungsbedarf gibt, damit möglicher Schaden vom Patienten abgewendet werden kann [4;5]. Machtgefälle und Autoritätsgradienten zwischen und innerhalb einer Berufsgruppe erschweren das "Speakingup" bezüglich der Patientensicherheit [6]. Zudem ist Speaking-up gegenüber Kollegen und Vorgesetzten häufig von der Sorge begleitet, soziale Beziehungen am Arbeitsplatz negativ zu beeinträchtigen. Mitarbeitende sind häufig nicht überzeugt, dass ihr Einspruch erwünscht ist und der Nutzen das persönliche Risiko rechtfertigt [7]. In einem stillen Abwägungsprozess, der zudem häufig sehr schnell erfolgen muss, wird das Risiko dann häufig höher gewertet als der Nutzen des Speaking-up [5]. Bedenken und Einwände werden verschwiegen. 
84 Insgesamt existieren im deutschsprachigen Raum bislang nur sehr vereinzelt Daten über das

85 Speaking-up von Mitarbeitenden in Gesundheitsinstitutionen. Die vorliegenden

Untersuchungen stammen zudem fast ausschliesslich aus der invasiven Medizin, z.B. anästhesiologischen Teams, und wurden mehrheitlich im Kontext von Simulationstrainings generiert [6;8;9]. Gegenstand unserer Arbeit war das Speaking-up Verhalten von Mitarbeitenden in der Onkologie. Die Onkologie ist ein multiprofessionell geprägter und hochkomplexer Bereich der Gesundheitsversorgung, in dem schon kleine Fehler zu schwerwiegenden Schädigungen führen können. Verschiedene medizinische Disziplinen und Professionen arbeiten eng zusammen, die Therapien sind häufig sehr komplex und die verwendeten Arzneimittel haben einen engen therapeutischen Korridor und erhebliches Gefahrenpotential. In der Onkologie ist eine offene, interprofessionelle Kommunikation auch hinsichtlich Sicherheitsaspekten besonders wichtig. Mithilfe einer schriftlichen Befragung von Pflegefachpersonen und Ärztinnen und Ärzten untersuchten wir, wie häufig Sicherheitsbedenken vorkommen, wie häufig diese angesprochen bzw. verschwiegen werden und welche Herausforderungen und Barrieren dem Speaking-up entgegenstehen. Da wir vermuteten, dass die Speaking-up Erfahrungen massgeblich von der eigenen hierarchischen Position beeinflusst sind, untersuchten wir Gruppenunterschiede zwischen Personen mit und ohne Leitungsfunktion und zwischen verschiedenen Berufsgruppen. Mithilfe von Fallgeschichten simulierten wir typische Speaking-up Situationen und erfassten, wie die befragten Personen sich verhalten würden und welche Faktoren das Speaking-up beeinflussen.

\section{Methode}

2.1. Design

Die explorative Studie beinhaltete 32 qualitative Interviews mit Ärztinnen, Ärzten und Pflegefachpersonen, die in der onkologischen Patientenversorgung tätig sind (Phase 1). In den persönlichen, semi-strukturierten Interviews wurden Fachpersonen verschiedener

111 Hierarchiestufen zu ihren persönlichen Erfahrungen und Erlebnissen befragt. Diese Interviews 
112 stellten neben der Literatur [4] eine wesentliche Grundlage dar, auf der dann ein schriftliches,

113 quantitatives Fragebogeninstrumente entwickelt wurde. An dieser schriftlichen Befragung

114 (Phase 2, Gegenstand der vorliegenden Arbeit) beteiligten sich neun onkologische Abteilungen von deutsch-schweizer Spitälern. Darunter waren Universitäts-, Kantons- und Regionalspitäler, stationäre und ambulante Abteilungen sowie pädiatrische onkologische Kliniken. Zur Rekrutierung der Spitäler wurden konsekutiv alle onkologischen Abteilungen für eine

118 Teilnahme angefragt bis so viele Mitarbeiter eingeschlossen waren, dass die geplante

119 Stichprobengrösse erreicht werden konnte ( $\mathrm{n}=$ mindestens 700 rückgesandte Fragebogen).

120 Zudem beteiligten sich die Mitglieder des Fachverbandes der Onkologiepflege. Die Studie wurde von der zuständigen Ethikkommission geprüft und für nicht bewilligungspflichtig und aus ethischer Sicht als unbedenklich erklärt (KEK-StV-Nr. 58/13).

\subsection{Fragebogeninstrument}

Das Fragebogeninstrument beinhaltete zwei Teile: Im allgemeinen Teil wurden die Häufigkeit von Sicherheitsbedenken, eigene Verhaltensweisen, Einstellungen, Normen, sowie soziale Unterstützung und psychologische Sicherheit am Arbeitsplatz erfasst. Wenn möglich verwendeten wir bereits validierte Fragen/Skalen. So inkludierten wir die für die Gesundheitsversorgung adaptierte „employee silence scale“ [10] und einzelne Fragen zur Erfassung der sozialen Unterstützung und psychologischen Sicherheit am Arbeitsplatz [11-13]. Die Häufigkeit von Sicherheitsbedenken und das eigene Speaking-up Verhalten wurden anhand einer 5-stufigen Häufigkeitsskala (nie; selten; manchmal; häufig; sehr häufig) erhoben. Alle andere Fragen des allgemeinen Teils wurden mit einer 7-stufigen Likert-Zustimmungsskala gemessen. Der allgemeine Teil bestand insgesamt aus 34 Fragen. In der vorliegenden Analyse gehen wir auf Fragen aus den Bereichen Häufigkeit von Sicherheitsbedenken, Häufigkeit des Schweigens (employee silence scale), Speaking-up als Patientensicherheits-Anwaltschaft, sowie Herausforderungen beim Speaking-up ein. In einer Faktorenanalyse wurden diese Themen als wichtige, gut abgrenzbare Faktoren identifiziert [14]. 
140 Im zweiten Fragebogenteil wurden Vignetten („Fallgeschichten“) präsentiert, die von den

141 Teilnehmern anhand verschiedener Fragen zu beurteilen waren. Diesen Ansatz wählten wir, da

142 sich in den Interviews deutlich zeigte, wie wichtig klinische (z.B. Risiko für den betroffenen

143 Patienten) und soziale Kontextfaktoren (z.B. Hierarchie der beteiligten Fachpersonen) für das

144 Speak-up Verhalten sind. In den kurzen Fallgeschichten war jeweils eine hypothetische

145 Situation geschildert, in der ein Kollege einen Fehler macht oder eine Sicherheitsregel

146 missachtet. Diese Rahmenhandlungen basierten auf Erfahrungsberichten, die wir in den

147 Interviews erhalten hatten und beinhalteten beispielsweise eine ausgelassene Hände-

148 Desinfektion bei einer Wunduntersuchung, einen Verordnungsfehler und weitere Situationen. In

149 die Rahmenhandlungen waren jeweils Detailinformationen („Faktoren“) eingebettet, die in 150 einem voll-faktoriellen Versuchsplan systematisch manipuliert worden waren. So präparierten wir beispielsweise 8 Vignetten, die eine ausgelassene Hände-Desinfektion beschrieben, sich aber darin unterschieden, zu welcher Berufsgruppe die handelnde Person gehörte (Oberärztin vs. leitende Pflegefachfrau), ob die Patientin und ihre Angehörigen die Situation aufmerksam

154 beobachten (ja vs. nein) und ob sich der Vorfall während der Visite vor vielen oder wenigen anwesenden Kollegen, also vor Publikum (ja vs. nein), abspielt (siehe Abbildung 1). Diese Faktoren wurden in den Interviews und zum Teil der Literatur als wichtige Einflussfaktoren für die Speaking-up Entscheidung identifiziert. Die Befragten bewerten jeweils eine von acht verschiedenen Versionen der Fallgeschichte „ausgelassene Hände-Desinfektion“, die innen randomisiert zugeteilt wurde. Die Teilnehmer beurteilten die Fälle anhand eines Fragensets, zum Beispiel wie hoch sie das Schadenspotential für den Patienten einschätzen, wie sie sich persönlich als „Bystander“ in der Situation verhalten würden (simuliertes Verhalten) und wie unangenehm das Speaking-up in der Situation für sie wäre. Der Vorteil dieses experimentellen Vorgehens ist, dass der Effekt der integrierten Faktoren auf die Beurteilung der Vignette extrahiert werden kann. So kann zum Beispiel der Einfluss der Berufsgruppe der beschriebenen Personen auf das antizipierte eigene Verhalten quantifiziert werden. Die klinischen Fallvignetten wurden mit Experten aus der onkologischen Versorgung diskutiert und von diesen als realistisch und verständlich bewertet. Der gesamte Fragebogen wurde einem 
168 Pretest unterzogen und dem Feedback entsprechend überarbeitet. In der vorliegenden Arbeit

werden nur die Vignetten-Antworten analysiert, die sich auf die Rahmenhandlung der ausgelassenen Händedesinfektion beziehen.

\subsection{Datenanalyse}

Die Fragen des allgemeinen Teils wurden deskriptiv ausgewertet. Um Unterschiede zwischen Gruppen von Mitarbeitenden (Pflege vs. Ärzte; Personen mit vs. ohne Leitungsfunktion) zu untersuchen, wurden Odds Ratios berechnet sowie Chi2- und t-Tests angewendet. Eine Regressionsanalyse wurde durchgeführt, um den Einfluss der Vignetten-Merkmale auf die selbst-berichtete Bereitschaft zum Speak-up zu untersuchen. Alle Tests waren zweiseitig und als Signifikanzniveau wurde $p \leq 0.05$ festgelegt.

\section{Ergebnisse}

An der schriftlichen Befragung beteiligten sich 1'013 Ärztinnen, Ärzte und Pflegefachpersonen (Rücklauf: 65\%). Tabelle 1 zeigt die demographischen Daten der Stichprobe.

\subsection{Häufigkeit von Sicherheitsbedenken}

Die befragten Mitarbeiter der onkologischen Abteilungen erleben im Spitalalltag häufig Situationen, die bei innen Sicherheitsbedenken auslösen: Konkrete Bedenken zur Patientensicherheit auf ihrer Abteilung zu haben gaben 37\% ( $n=375)$ mit „manchmal“ und 16\% ( $n=159)$ mit „häufig“ oder „sehr häufig“ an. Von den Befragten berichteten 38\% ( $n=380)$, dass sie „manchmal“ einen Fehler bemerken, der, wenn er unbeachtet bleibt, gefährlich für den betroffenen Patienten wäre (13\% „häufig“ ( $n=128)$, 4\% ( $n=40)$ „sehr häufig“). Dass Kollegen sich bewusst oder unbewusst nicht an Regeln halten, die für die Patientensicherheit wichtig sein können (z.B. Doppelkontrollen oder Hygienerichtlinien) gaben 30\% ( $n=305)$ mit „manchmal“ an und 13\% ( $n=127)$ mit „häufig“ oder „sehr häufig“. Alle Arten von Sicherheitsbedenken wurden signifikant häufiger von Pflegefachpersonen (vs. Ärzten) und von Personen ohne Leitungsfunktion (vs. mit Leitungsfunktion) berichtet. So gaben von den 
Pflegefachpersonen 53\% an, dass sie nur selten/nie Verletzungen von Sicherheitsregeln durch

Kollegen beobachten während es von den Ärztinnen und Ärzten 68\% waren (OR=0.5, $\mathrm{p}<0.001)$.

\subsection{Speaking-up Verhalten}

Die meisten der Befragten kennen Situationen, in denen sie ihre Zweifel und Bedenken zur

Patientensicherheit nicht ausgesprochen haben. Die Antworten auf der „employee silence

scale" zeigen, dass das Zurückhalten von Verbesserungsvorschlägen und konkreten

Sicherheitsbedenken kein seltenes Verhalten ist (Tabelle 2). Beispielsweise berichten $29 \%$ der

Befragten, „manchmal“, „häufig“ oder „sehr häufig“ Gedanken oder Ideen zur Verbesserung der

Patientensicherheit in Ihrer Abteilung für sich zu behalten. Knapp 20\% gaben an, mindestens

„manchmal“ mögliche Sicherheitsprobleme in ihrer Abteilung festzustellen und anderen

Personen nichts darüber zu sagen. Wiederum berichten Pflegefachpersonen (vs. Ärzte) und

Personen ohne Leitungsfunktion (vs. mit Leitungsfunktion) deutlich häufiger, ihre

Sicherheitsbedenken nicht anzusprechen. Unter den Personen mit Leitungsfunktion haben 73\% noch nie Hinweise verschwiegen, die möglicherweise eine Gefahr für Patienten reduziert hätten, während es unter den Personen ohne Leitungsfunktion $60 \%$ waren (OR=1.8, $p \leq 0.001)$.

\subsection{Speaking-up als Patientensicherheits-Anwaltschaft}

215 Die befragten Fachpersonen fühlen sich in hohem Mass mit(-verantwortlich) für die

216 Patientensicherheit. Sie haben mehrheitlich eine positive Haltung zur Patientensicherheits-

217 Anwaltschaft (Tabelle 3). Das Eintreten für die Einhaltung von Sicherheitsregeln wird von der

218 weit überwiegenden Mehrheit der Fachpersonen als Teil ihrer professionellen Rolle verstanden.

219 Beispielsweise stimmten 84\% der Befragten zu, dass es ihre Aufgabe ist, auf die Einhaltung von Sicherheitsregeln zu achten. Allerdings zeigen sich signifikante Unterschiede auf dem

221 Skalenmittelwert zwischen Personen mit vs. ohne Leitungsfunktion (6.3 vs. 6.0, $p \leq 0.001)$ sowie

222 Ärzten und Pflegefachpersonen (6.2 vs. 6.0, p $\leq 0.01$ ). Das heisst, dass sich Personen mit 
223

224

225

226

227

228

229

230

231

232

233

234

235

236

237

238

239

240

241

242

243

244

245

246

247

Leitungsfunktion eher als Anwalt für die Patientensicherheit fühlen und sich für die Einhaltung von Regeln einsetzen.

\subsection{Anforderungen und Herausforderungen beim Speaking-up}

Die Anforderungen an und das Umsetzen von Speaking-up empfindet ein erheblicher Teil der Befragten als schwierig und herausfordernd (Tabelle 4). Dies betrifft sowohl die Entscheidung, ob und wie man erfahrene Kollegen auf einen Fehler hinweisen kann als auch auf die richtige Form. Beispielsweise stimmen zwei Drittel der Befragten zu, dass man ein feines Gespür benötigt, um einen Vorgesetzten auf eine heikle Situation hinzuweisen. Etwa die Hälfte der Fachpersonen findet es schwierig, die richtige Art und Weise zu finden, um Sicherheitsbedenken anzusprechen, und 38\% bestätigt, dass es Beharrlichkeit braucht, bis man mit Sicherheitsbedenken durchdringt. Aber auch Resignation und Frustration sind verbreitete Phänomene im Kontext des Speaking up. Die Hälfte der Befragten findet es frustrierend, immer auf die gleichen Sicherheitsregeln hinweisen zu müssen und ein Viertel resigniert, weil das Speaking-up im Effekt erfolglos bleibt, sich also nichts ändert, wenn Sicherheitsbedenken und Einwände angesprochen werden. Die wahrgenommenen Herausforderungen unterscheiden sich nicht wesentlich zwischen Personen mit vs. ohne Leitungsfunktion (Skalenmittelwert 4.1 vs. 4.2, $\mathrm{p}=0.09$ ). Allerdings weisen Pflegefachpersonen

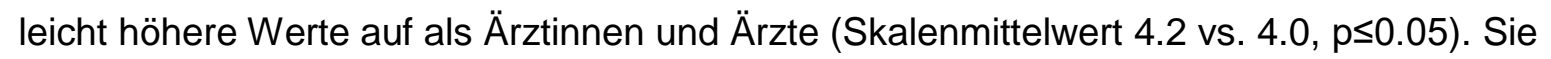
finden das Ansprechen von Sicherheitsbedenken also herausfordernder und frustrierender als die ärztlichen Mitarbeitenden.

\subsection{Bewertung von Vignetten und simuliertes Speaking-up Verhalten}

Die Bewertung der acht verschiedenen Hände-Desinfektions-Vignetten gibt Aufschluss darüber, wie die Fachpersonen das Schadenspotential einschätzen, wie schwierig sie das Speaking-up in einer solchen typischen Situation finden, wie sie sich hypothetisch verhalten würden und welche Faktoren diese Bewertungen beeinflussen. Über alle Vignetten-Variationen hinweg gaben ein Drittel der Befragten (32\%) an, dass sie den in der Vignette beschriebenen 
Kollegen nicht auf die ausgelassene Hände-Desinfektion bei einer frisch-operierten

onkologischen Patientin hinweisen würden. Der Anteil der Befragten, die nichts sagen würden, war am höchsten für den Fall, dass ein Oberarzt die Hände-Desinfektion vergisst und die Patientin die Situation aufmerksam verfolgt (48\% würden es vorziehen, zu schweigen). Hingegen würden sich in der gleichen Situation $76 \%$ für das Speaking-up entscheiden, wenn es eine Pflegefachfrau ist, die die Händehygiene vergisst. Die Bewertungen der Teilnehmenden sind nicht nur durch die Merkmale der Vignette beeinflusst, sondern auch durch den eigenen hierarchischen Status. Abbildung 2 zeigt, dass sich zwar die Einschätzung des Schadenspotentials nicht zwischen Personen mit vs. ohne Leitungsfunktion unterscheidet. Allerdings ist der wahrgenommene Diskomfort mit dem Speaking-up erheblich höher bei Personen ohne vs. mit Leitungsfunktion (4.0 vs. $3.3, p<0.001)$ und auch das antizipierte, eigene Speaking-up Verhalten ist höher bei Leitungspersonen (5.7 vs. 5.0, $\mathrm{p}<0.001$ ). Eine Regressionsanalyse zeigt, dass auf Ebene der Vignetten-Merkmale die Berufsgruppe des Handelnden sowie die Aufmerksamkeit der Patientin für die Situation wichtige Prädiktoren für das antizipierte Speaking-up Verhalten des Befragten sind. Auf Ebene der Bewertung der Situation sind es das Schadenspotential für die Patientin und der empfundene eigene Diskomfort mit dem Speaking-up, die wesentliche Einflussfaktoren sind. Auf Ebene des Befragten sind es das Geschlecht, das Alter und die Berufsgruppe, die das hypothetische Speaking-up beeinflussen. Insgesamt kann das Regressionsmodell einen substantiellen Teil der Varianz im antizipierten Speaking-up Verhalten erklären (Bestimmtheitsmass $R^{2}=0.31$; Cohens $\left.\mathrm{f}^{2}=0.46\right)$.

\section{Diskussion}

Unsere Studie bestätigt, dass ein erheblicher Teil der Mitarbeitenden im Krankenhaus regelmässig konkrete Bedenken hinsichtlich der Patientensicherheit hat. Viele Mitarbeitenden halten diese Bedenken, Zweifel, Fragen, Sorgen, aber auch Verbesserungsvorschläge zurück und sprechen diese auf ihren Abteilungen nicht an. Damit geht ein überaus wichtiges Potential zur Prävention von Schädigungen und für systematische Fortschritte in der Patientensicherheit 
279

280

281

282

283

284

285

286

287

288

289

290

291

292

293

294

295

296

297

298

299

300

301

302

303

304

305

verloren. Es ist für alle Beteiligten besonders tragisch, wenn ein Patient Schaden nimmt, obwohl „jemand im Raum war“, der ahnte oder wusste, dass etwas falsch läuft. Ein häufiger Anlass für das Zurückhalten von Bedenken ist die Verletzung von Sicherheitsregeln durch Kollegen, wie beispielsweise das Auslassen der Händedesinfektion oder übersprungene Vorsichtsmassnahmen bei isolierten Patienten. In unserer Stichprobe beobachteten $43 \%$ der Teilnehmer solche Regelverletzungen auf ihrer Abteilung mindestens „manchmal“. Aus unseren Interviews wissen wir, dass das Speaking-up in solchen Situationen häufig nicht zum Erfolg - der Einhaltung einer Regel - führt, sondern unwirksam bleibt. Diese Erfahrung wiederum wird bei den Mitarbeitenden sehr schnell dazu führen, dass sie bei einer erneuten Beobachtung schweigen werden. Werden Regelverletzungen wiederholt nicht angesprochen sondern stillschweigend toleriert, kann es zu einer „Normalisierung der Abweichung“ und einer Erosion von Sicherheitsstandards kommen [15]. Auch in der Befragung zeigte sich ein erhebliches Frustrations- und Resignationspotential. Die Hälfte der Mitarbeitenden gibt an, dass es frustrierend ist, immer auf die gleichen Sicherheitsregeln hinweisen zu müssen und ein Viertel resigniert, weil das Speaking-up ineffektiv bleibt.

Unsere Daten geben auch deutliche Hinweise auf die Bedeutung des Autoritätsgradienten. Personen mit Leitungsfunktion erachten es eher als ihre Aufgabe sich für die Einhaltung von Regeln zu engagieren, auch wenn sie das Speaking-up selber ebenso als Herausforderung wahrnehmen. Die Analyse der Vignetten-Beurteilungen zeigt ein interessantes Muster: In der Einschätzung des Schadenspotentials der Situation zeigte sich kein Zusammenhang mit einer Leitungsfunktion. Das simulierte Speaking-up Verhalten und das damit verbundene Unbehagen hingegen variiert wesentlich je nach hierarchischem Status. Das heisst, Unterschiede im Speaking-up Verhalten sind nicht auf eine ungleiche Risikobewertungen zurückzuführen (z.B. weil Leitungspersonen besser qualifiziert wären, Risiken zu erkennen) sondern tatsächlich auf die soziale Konstellation und die persönliche Abwägungsentscheidung. 
306 Wenn also viele Mitarbeiter Risiken und Regelverletzungen erkennen, mehrheitlich auch

307 anerkennen, dass die Patientensicherheits-Anwaltschaft Teil ihrer Rolle ist und Patienten vom

308 Speaking-up profitieren, aber in der Umsetzung des Speaking-up oftmals scheitern, ergeben

309 sich daraus ein Handlungsbedarf und Fragen, wie man die Mitarbeitenden unterstützen und

310 ermutigen kann. In den vorgängig zu dieser Befragung durchgeführten Interviews zeigte sich

311 sehr deutlich, dass typische Speaking-up Situationen häufig schnell und unerwartet eintreten.

312 Die Mitarbeitenden sind meist nicht darauf vorbreitet. Sie versuchen, die richtigen Worte zu

313 finden um einerseits das Risiko für den Patienten abzuwenden und gleichzeitig keine sozialen

314 „Kollateral-Schäden“ zwischen sich und ihren Kollegen zu produzieren [16]. Einerseits ist es

315 wichtig, in Teams konkrete akzeptable Formulierungen vorzuschlagen oder sogar verbindlich

316 zu verabreden, insbesondere für Situationen, in denen Patienten anwesend sind und der

317 Abwägungskonflikt dadurch weiter verschärft ist. Die Stiftung Patientensicherheit Schweiz hat

318 ein Manual entwickelt, das Mitarbeitende, Führungspersonen und Organisationen in der

319 Umsetzung unterstützen soll und das praktische Empfehlungen und eine „Speaking-up Pocket

320 Karte" enthält [17]. Der Effekt von Trainings und Schulungen zur Förderungen des Speak-up

321 Verhaltens ist bislang unklar. Beispielsweise zeigten Raemer et al., das ein 50-minütiger

322 Workshop keinen Einfluss auf das Speaking-up Verhalten von Anästhesisten in

323 entsprechenden Simulations-Szenarien hatte [9]. Andere Studien kamen zu positiveren

324 Ergebnissen [18;19]. Andererseits scheint es wichtig, dass in Krankenhäusern konsequent,

325 systematisch und redundant von der Führung das Speaking-up gefördert und bekräftigt wird.

326 Gerade Kultur und Verhalten der direkten Vorgesetzen hinsichtlich Aufgeschlossenheit und

327 Integration haben einen wesentlichen Effekt auf die von den Mitarbeitern empfundene

328 psychologische Sicherheit am Arbeitsplatz, die wiederum ein wesentlicher Prädiktor für das

329 Speaking-up und das Mitarbeiter-Engagement für Qualitätsverbesserungen ist [14;20].

330

\section{Schlussfolgerungen}

332 Unsere Studie liefert erste Daten und Erkenntnisse über die Häufigkeit und das Zurückhalten 333 von Sicherheitsbedenken und Erfahrungen mit dem Speaking-up im deutschsprachigen Raum 
334 und speziell in der Onkologie. Mitarbeitende im Spital haben häufig Sicherheitsbedenken, 335 sprechen diese aber oft nicht an. Dadurch geht ein wichtiges Potential zur Verbesserung der 336 Patientensicherheit verloren. Die Entwicklung von Interventionen zur Förderung der Speaking337 up Kultur ist eine wichtige zukünftige Aufgabe um diese Ressource zu nutzen.

338

339

340 


\section{Referenzen}

(1) Leonard M, Graham S, Bonacum D. The human factor: the critical importance of effective teamwork and communication in providing safe care. Qual Saf Health Care 2004;13:i85-i90.

(2) Maxfield DG, Lyndon A, Kennedy HP, O'Keeffe DF, Zlatnik MG. Confronting safety gaps across labor and delivery teams. Am J Obstet Gynecol 2013;209:402-8.

(3) Maxfield D, Grenny J, Lavandero R, Groah L. The silent treatment. Why safety tools and checklists aren't enough to save lives. Patient Safety and Quality Healthcare 2011;September/October.

(4) Okuyama A, Wagner C, Bijnen B. Speaking up for patient safety by hospital-based health care professionals: A literature review. BMC Health Serv Res 2014;14:61-9.

(5) Schwappach D, Gehring K. Trade-offs between voice and silence: a qualitative exploration of oncology staff's decisions to speak up about safety concerns. BMC Health Serv Res 2014;14:303.

(6) St.Pierre M, Scholler A, Strembski D, Breuer G. Äussern Assistenzärzte und Pflegekräfte sicherheitsrelevante Bedenken? Anaesthesist 2012;61:857-66.

(7) Edmondson AC. Speaking up in the operating room: How team leaders promote learning in interdisciplinary action teams. Journal of Management Studies 2003;40:1419-52.

(8) Kolbe M, Burtscher MJ, Wacker J, Grande B, Nohynkova R, Manser T, et al. Speaking up is related to better team performance in simulated anesthesia inductions: An observational study. Anesthesia \& Analgesia 2012;115:1099-108.

(9) Raemer DB, Kolbe M, Minehart RD, Rudolph JW, Pian-Smith MCM. Improving Anesthesiologists' Ability to Speak Up in the Operating Room: A Randomized Controlled Experiment of a Simulation-Based Intervention and a Qualitative Analysis of Hurdles and Enablers. Acad Med 2016;91:530-9.

(10) Tangirala S, Ramanujam R. Employee silence on critical work issues: The cross level effects of procedural justice climate. Personnel Psychology 2008;61:37-68.

(11) Prümper J, Hartmannsgruber K, Frese M. KFZA. Kurzfragebogen zur Arbeitsanalyse. Zeitschrift für Arbeits- und Organisationspsychologie 1995;39:125-32.

(12) Baer M, Frese M. Innovation is not enough: Climates for initiative and psychological safety, process innovations, and firm performance. J Organiz Behav 2003;24:45-68.

(13) Edmondson A. Psychological safety and learning behavior in work teams. Administrative Science Quarterly 1999;44:350-83.

(14) Schwappach DLB, Gehring K. Frequency of and predictors for withholding patient safety concerns among oncology staff: A survey study. Eur J Cancer Care 2015;24:395-403.

(15) Price MR, Williams TC. When Doing Wrong Feels So Right: Normalization of Deviance. $J$ Pat Saf 9000; Publish Ahead of Print.

(16) Schwappach DLB, Gehring K. 'Saying it without words': A qualitative study of oncology staff's experiences with speaking up about safety concerns. BMJ Open 2014;4:e004740. 


\section{Danksagung}

Wir danken allen Teilnehmern an unserer Studie für die Beantwortung des Fragebogens.

401

403 Die Studie wurde durch die Forschungsförderung der Krebsforschung Schweiz (KFS-2974-08404 2012) und die Hanela-Stiftung finanziert.
(17) Gehring K, Schwappach D. Wenn Schweigen gefährlich ist: Speak Up für mehr Sicherheit in der Patientenversorgung. Zürich: Stiftung für Patientensicherheit Schweiz; 2015.

(18) Sayre MM, McNeese-Smith D, Leach LS, Phillips LR. An Educational Intervention to Increase 'Speaking-Up' Behaviors in Nurses and Improve Patient Safety. J Nurs Care Qual 2012;27:154-60.

(19) Pian-Smith MCM, Simon R, Minehart RD, Podraza M, Rudolph J, Walzer T, et al. Teaching residents the two-challenge rule: A simulation-based approach to improve education and patient safety. Simul Healthc 2009;4:84-91.

(20) Nembhard IM, Edmondson AC. Making it safe: the effects of leader inclusiveness and professional status on psychological safety and improvement efforts in health care teams. J Organiz Behav 2006;27:941-66.

\section{Interessenkonflikte}

Der Autor erklärt keine Interessenskonflikte.

\section{$402 \quad$ Finanzierung}


Tabelle 1. Demografische Angaben zur Stichprobe (n=1’013)

406

\begin{tabular}{cc}
\hline Merkmal & Teilnehmer \\
\hline
\end{tabular}

Geschlecht

Frauen

$800 \quad 80$

Männer

21320

Alter, Durchschnitt (SD) in Jahren

40 (11)

18-25 Jahre

$94 \quad 9$

26-40 Jahre

$441 \quad 44$

41-55 Jahre

$394 \quad 39$

56-65 Jahre

$\begin{array}{ll}73 & 7\end{array}$

Beruf

Arzt/Ärztin

$131 \quad 13$

Assistenzarzt/Ärztin

Oberarzt/ Ärztin

616

Leitender Arzt/ Ärztin

$38 \quad 4$

Chefarzt/ Ärztin

232

91

Pflegefachperson

$780 \quad 79$

Pflegefachperson in Ausbildung

$22 \quad 2$

Pflegefachperson

$570 \quad 58$

Leitende Pflegefachperson

$151 \quad 15$

Pflegeexperte/Expertin

$37 \quad 4$

Andere (z.B. Apotheker/ln)

$71 \quad 7$

Tätigkeit mit Leitungsfunktion

$221 \quad 22$

Tätigkeit in der Onkologie, Durchschnitt (SD) in Jahren

$9(7)$

1-5 Jahre

$359 \quad 38$

6-10 Jahre

$266 \quad 28$

11-25 Jahre

$290 \quad 31$

>25 Jahre

$26 \quad 3$

407 
408 Tabelle 2. Zurückhalten von Sicherheitsbedenken („employee silence scale“, adaptiert nach 409 [10])

\begin{tabular}{|c|c|c|c|c|}
\hline Wie häufig ... & Nie & Selten & Manchmal & $\begin{array}{l}\text { (Sehr) } \\
\text { häufig }\end{array}$ \\
\hline ... haben Sie entschieden, Ihre Bedenken zur & 300 & 388 & 239 & 80 \\
\hline Patientensicherheit nicht zu äussern? & $(30 \%)$ & $(39 \%)$ & $(24 \%)$ & $(8 \%)$ \\
\hline $\begin{array}{l}\text {... haben Sie Gedanken oder Ideen zur } \\
\text { Verbesserung der Patientensicherheit in Ihrer } \\
\text { Abteilung für sich behalten? }\end{array}$ & $\begin{array}{c}325 \\
(32 \%)\end{array}$ & $\begin{array}{c}393 \\
(39 \%)\end{array}$ & $\begin{array}{c}191 \\
(19 \%)\end{array}$ & $\begin{array}{c}97 \\
(10 \%)\end{array}$ \\
\hline $\begin{array}{l}\text {... haben Sie mögliche Sicherheitsprobleme in Ihrer } \\
\text { Abteilung festgestellt und anderen Personen nichts } \\
\text { darüber gesagt? }\end{array}$ & $\begin{array}{c}497 \\
(50 \%)\end{array}$ & $\begin{array}{c}324 \\
(32 \%)\end{array}$ & $\begin{array}{c}136 \\
(14 \%)\end{array}$ & $\begin{array}{c}46 \\
(5 \%)\end{array}$ \\
\hline $\begin{array}{l}\text {... hatten Sie Fragen zur Patientensicherheit in Ihrer } \\
\text { Abteilung und haben diese lieber nicht gestellt? }\end{array}$ & $\begin{array}{c}482 \\
(48 \%)\end{array}$ & $\begin{array}{c}345 \\
(34 \%)\end{array}$ & $\begin{array}{c}93 \\
(9 \%)\end{array}$ & $\begin{array}{c}86 \\
(9 \%)\end{array}$ \\
\hline $\begin{array}{l}\text {... haben Sie geschwiegen, obwohl Ihre Hinweise } \\
\text { möglicherweise eine Gefahr für Patienten reduziert } \\
\text { hätten? }\end{array}$ & $\begin{array}{c}629 \\
(63 \%)\end{array}$ & $\begin{array}{c}312 \\
(31 \%)\end{array}$ & $\begin{array}{c}53 \\
(5 \%)\end{array}$ & $\begin{array}{c}8 \\
(1 \%)\end{array}$ \\
\hline
\end{tabular}


411 Tabelle 3. Speaking-up als Patientensicherheits-Anwaltschaft, gemessen auf einer 7-Punkt

412 Likert Skala.

Fragebogen-item Zustimmung*

(\%)

Mittelwert (SD)

\begin{tabular}{lcc}
\hline Es gibt Sicherheitsmassnahmen, auf deren Einhaltung & 910 \\
$\begin{array}{l}\text { ich auch gegenüber einem ranghöheren Kollegen } \\
\text { beharren würde. }\end{array}$ & $(91 \%)$ & $6.2(1.4)$
\end{tabular}

Hier fühlt sich jeder für die Sicherheit der Patienten verantwortlich. 857

Unsere Patienten profitieren davon, dass wir $(85 \%)$

Mitarbeitenden uns gegenseitig auf 914

Sicherheitsbedenken ansprechen.

Es ist meine Aufgabe, auf die Einhaltung von 851

Sicherheitsregeln zu achten. (84\%)

$6.0(1.6)$

Skala (Cronbach Alpha=0.69) $--$ $6.1(1.0)$

* Dichotomisiert mit $x>4$ = Zustimmung 
414 Tabelle 4: Anforderungen und Herausforderungen beim Speaking-up, gemessen auf einer 7-

415 Punkt Likert Skala.

\begin{tabular}{lcl}
\hline Fragebogen-item & Zustimmung $^{*}$ & Mittelwert (SD) \\
$\%$ &
\end{tabular}

Man braucht ein feines Gespür, um einen Vorgesetzten auf seinen möglichen Fehler oder eine heikle Situation 691 hinzuweisen.

$(69 \%) \quad 5.0(1.6)$

\begin{tabular}{lcc}
\hline $\begin{array}{l}\text { Es ist frustrierend, immer auf die gleichen } \\
\text { Sicherheitsregeln hinweisen zu müssen. }\end{array}$ & $\begin{array}{c}520 \\
(52 \%)\end{array}$ & $4.5(1.8)$ \\
\hline $\begin{array}{l}\text { Es ist schwierig, die richtige Art und Weise zu finden, } \\
\text { um Sicherheitsbedenken anzusprechen. }\end{array}$ & $\begin{array}{c}489 \\
(49 \%)\end{array}$ & $4.2(1.8)$ \\
\hline Manchmal resigniere ich, weil sich nichts ändert, wenn & 254 & $3.2(1.9)$ \\
man seine Einwände zur Patientensicherheit vorbringt. & $(26 \%)$ & \\
\hline $\begin{array}{l}\text { Die Entscheidung, ob und wie ich einen erfahrenen } \\
\text { Kollegen auf einen Fehler hinweise, ist manchmal sehr }\end{array}$ & 543 & $4.3(1.8)$ \\
schwierig. & $(54 \%)$ & \\
\hline Manchmal erfordert es viel Beharrlichkeit, bis ich mit & 376 & $4.0(1.7)$ \\
konkreten Bedenken zur Sicherheit eines Patienten & $(38 \%)$ & \\
durchdringe. & $--2(1.1)$ \\
\hline Skala (Cronbach Alpha=0.71) & \\
\hline * Dichotomisiert mit x>4 = Zustimmung & \\
\hline
\end{tabular}


417 Abbildung 1: Beispiel-Vignette aus der Befragung.

418 Legende: Die roten Auszeichnungen signalisieren Faktoren, die experimentell variiert wurden.

419 Beispielsweise wurde „Oberärztin“ in anderen Vignetten durch „leitende Pflegefachperson“

420 ersetzt.

\section{Beispiel Vignette (B-8, Set 1)}

Sie sind mit mehreren Ärzten und Pflegefachpersonen auf Visite bei einer onkologischen Patientin. Die Oberärztin will die Wunde der frisch-operierten Patientin untersuchen. Die Patientin und ihre Eltern verfolgen alles aufmerksam. Die Oberärztin benutzt keine Handschuhe und hat auch die 421 Hände nicht neu desinfiziert. 
424 Abbildung 2: Beurteilung des Schadenspotentials, der Wahrscheinlichkeit für Speaking-Up 425 und des mit Speaking-up verbundenen Unbehagens nach Leitungsfunktion

426 Legende: Mittelwert-Berechnung über alle Variationen der Vignette „ausgelassene Hände-

427 Desinfektion“ hinweg, gemessen auf einer 7-Punkt Likert Skala.

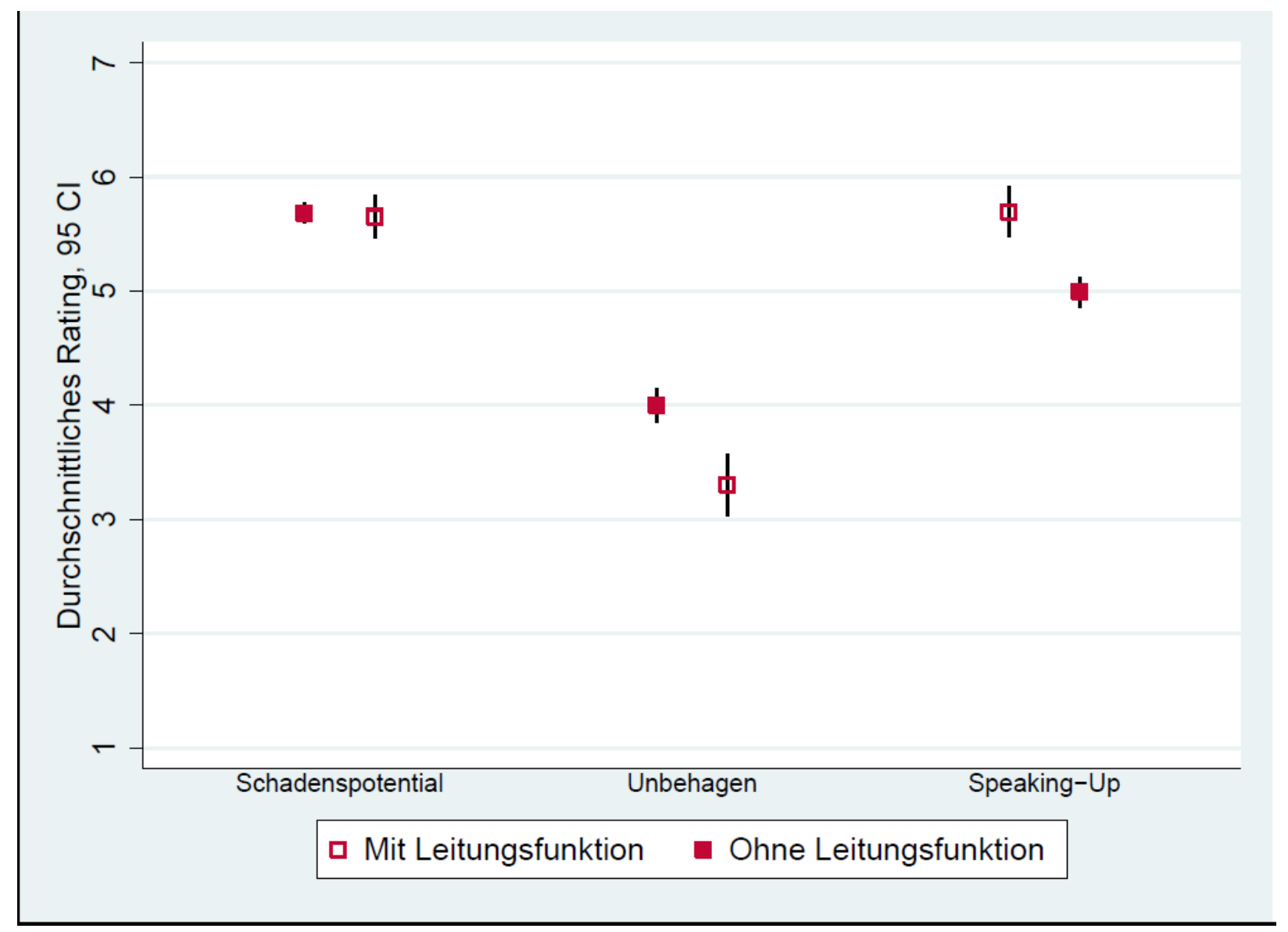

